\title{
La photographie de la Palestine au temps du collodion : une spécificité britannique?
}

Picturing Palestine in the collodion era: a British stance?

Palästina-Bilder in der Ära des Kollodiums: eine britische Besonderheit?

Daniel Foliard

\section{(2) OpenEdition \\ Journals}

Édition électronique

URL : http://journals.openedition.org/rh19/4351

DOI : 10.4000/rh19.4351

ISSN : $1777-5329$

Éditeur

La Société de 1848

Édition imprimée

Date de publication : 31 décembre 2012

Pagination : 161-183

ISSN : 1265-1354

Référence électronique

Daniel Foliard, « La photographie de la Palestine au temps du collodion : une spécificité britannique ?», Revue d'histoire du XIXe siècle [En ligne], 45 | 2012, mis en ligne le 31 décembre 2015, consulté le 20 avril 2019. URL : http://journals.openedition.org/rh19/4351 ; DOI : 10.4000/rh19.4351 


\section{DANIEL FOLIARD}

\section{La photographie de la Palestine au temps du collodion : une spécificité britannique?}

Dans l'un des premiers ouvrages à tenter une comparaison des œuvres des premiers photographes qui parcourent la Palestine à partir de la seconde moitié des années 1840, Yeshayahu Nir évoque l'existence d'une ligne de partage entre catholiques français et protestants britanniques ${ }^{2}$. Le choix des lieux fixés par la photographie, comme les compositions, révèle d'après lui une multiplicité de visions, souvent inspirées par des héritages culturels très variables en fonction des origines nationales. Plus récemment et dans un tout autre domaine, des travaux sur les missions au Proche-Orient sont venus confirmer l'intérêt d'une approche qui prenne en compte le rôle des rivalités nationales et interconfessionnelles dans la définition des projets de conversion en Palestine et dans les espaces voisins $s^{3}$. Il convient, bien sûr, de manier avec précaution l'idée que l'origine nationale ou la confession puissent avoir déterminé les pratiques des pionniers de la photographie et le regard du voyageur face à l'Orient biblique. Être un photographe français n'oblige en rien à adhérer à une lecture purement catholique du paysage palestinien. Être britannique c'est aussi, et avant tout, être anglais, écossais ou gallois : le concept même de nation britannique pose des difficultés jusqu'à nos jours ${ }^{4}$.

1. La technique du collodion humide est inventée en 1850 par Gustave le Gray puis améliorée par Frederick Scott Archer. Après dissolution de nitrocellulose dans un mélange d'éther et d'alcool, le photographe plonge une plaque de verre dans le liquide. Au contact de l'air, les solvants s'évaporent et laissent un film qu'il faut sensibiliser dans du nitrate d'argent. Le développement n'exige pas moins de manipulation puisqu'il faut plonger la plaque dans de l'acide gallique ou du sulfate de fer puis fixer l'image dans du cyanure de potassium. Le procédé est utilisé pendant une trentaine d'années avant d'être supplanté par les plaques au gélatino-bromure d'argent dans les années 1870 puis par le film photographique de George Eastman à partir de 1884 .

2. Yeshayahu Nir, The Bible and the image: the history of photography in the Holy Land, 1839-1899, Philadelphie, University of Pennsylvania Press, 1985, p. 86-107 et Yeshayahu Nir, "Cultural Predispositions in Early Photography: The Case of the Holy Land”, Journal of communication, XXXV, 3 (1985), p. 31-50. Nissan Perez (Focus East: early photography in the Near East (1839-1885), New York, Abrams, 1988, p. 81-85) propose aussi une comparaison des attitudes françaises et britanniques, mais elle repose sur des stéréotypes assez éculés sur les Français supposément exubérants et les Britanniques introvertis. 3. Friedrich, Norbert, Uwe Kaminsky et Roland Löffler [dir.], The social dimension of Christian missions in the Middle East: historical studies of the 19th and 20th centuries, Stuttgart, Steiner, 2010.

4. Cf. Keith Robbins, Nineteenth-century Britain: England, Scotland, and Wales: the making of a nation, Oxford, Oxford University Press, 1989. Il ne faut pas non plus oublier le rôle joué par les Irlandais dans l'exploration de l'Orient comme le montre Haim Goren, Dead Sea level: science, exploration and imperial 
La Palestine est toutefois un territoire à part dans les regards européens, particulièrement révélateur des prédispositions culturelles de l'observateur. De ce point de vue, le XIX siècle est déterminant. À l'instar du reste de l'Empire ottoman, la Terre Sainte devient un enjeu pour les Puissances dans le cadre plus général de la Question d'Orient ${ }^{5}$. Son ouverture progressive aux voyageurs occidentaux favorise un dévoilement souvent marqué par les contextes nationaux ${ }^{6}$. Une mise en regard des photographies d'Orient, déjà amorcée par Elias Sanbar ou par l'exposition Voyage en Orient, mérite d'être approfondie en prenant en compte la diversité des représentations européennes de la Terre Sainte.

À défaut de pouvoir envisager une démarche systématique de comparaison, qui mériterait un ouvrage, c'est la photographie britannique de la Palestine, très dynamique dans cette région entre la fin des années 1840 et le début des années 1870, qui est envisagée ici . En considérant l'œuvre d'un praticien en particulier, Frank Mason Good, on propose d'évoquer les spécificités de la photographie de la Terre Sainte par les Britanniques ainsi que ses usages et sa diffusion ${ }^{8}$. S'appuyer sur le travail de ce photographe en particulier

interests in the Near East, Londres, I.B. Tauris, 2011, p. 273-274. Utiliser l'adjectif britannique pour traiter de l'image de l'Orient au XIX ${ }^{e}$ ne semble toutefois pas usurpé comme l'a montré la récente exposition The Lure of the East: British Orientalist Painting (Londres, Tate Britain, du 4 juin au 31 août 2008). 5. Cf. Henry Laurens, La question de Palestine. 1799-1922, linvention de la terre sainte, Paris, Fayard, 1999, tome 1.

6. Une approche statistique des nationalités des photographes actifs en Orient au XIX ${ }^{\mathrm{e}}$ siècle ( $c f$. Nissan Perez, Focus East..., op.cit., p. 76) montre que les deux nations les plus représentées sont la France et la Grande-Bretagne. On ne saurait négliger le rôle des photographes et orientalistes d'autres nationalités. Au sujet de l'orientalisme allemand, $c f$. la série d'articles publiée dans Comparative Studies of South Asia, Africa and the Middle East, XXIV, 2 (2004), p. 97-180. Pour la Scandinavie, $c f$. Elisabeth Oxfeldt, Nordic orientalism: Paris and the cosmopolitan imagination 1800-1900, Copenhagen, Museum Tusculanum Press, 2005.

7. Après l'ère des daguerréotypistes, où les photographes français furent particulièrement actifs, Horace Vernet, Frédéric Goupil-Fesquet, Joseph-Philibert Girault de Prangey ou Maxime du Camp, la production de négatifs sur plaque de collodion humide fut commercialement dominée par des Britanniques. Dès la guerre de Crimée, Roger Fenton et James Robertson couvrent pour la première fois un conflit armé par l'image argentique. Le révérend George Wilson Bridges part de 1856 à 1860 en calotypiste. Dès 1857, l'entreprenant Francis Frith photographie l'Égypte et la Palestine. Deux ans plus tard, les tirages du révérend James Graham, actif à Jérusalem depuis 1853, sont exposés à Paris par la société française de photographie. L'Écossais John Cramb part en 1860 à la demande de l'éditeur William Collins pour réaliser des centaines de photographies de la Terre Saine. Les tirages luxueux de Francis Bedford, qui accompagne le Prince de Galles dans son voyage en Orient en 1862, témoignent à leur tour de la vivacité de l'activité des photographes britanniques en Palestine peu avant l'ouverture du canal de Suez. À cette liste, incomplète, il faut aussi ajouter les travaux de James McDonald, employé par le Palestine Exploration Fund ( $c f$. infra). À propos des liens entre la culture britannique et la Palestine, $c f$. Eitan Bar-Yosef, The Holy Land in English culture 1799-1917: Palestine and the question of Orientalism, Oxford, Clarendon Press, 2005.

8.Frank Mason Good n'a pas encore fait l'objet d'une monographie, à la différence de Francis Frith (Nickel Douglas, Francis Frith in Egypt and Palestine: a Victorian photographer abroad, Princeton, Princeton University Press, 2004). Il est régulièrement évoqué dans la littérature consacrée à la photographie orientaliste. $C f$. Oztuncay Bahattin, The Photographers of Constantinople: Text \& photographs, Istanbul, Aygaz, 2003, p. 92-93; Michael Bartram, The Pre-Raphaelite camera: aspects of Victorian photography, Boston, Little, Brown and Company, 1985, p. 97; Shimon Gibson, Jerusalem in original photographs, 1850-1920, London, Stacey International, 2003, p. 175; Badr Hajj, Romantic Lebanon: the European view 1700-1900, London, British Lebanese Association, 1986, p. 89; Yeshayahu Nir, The Bible and the image... op.cit., p. 141 ; Elias Sanbar, Les Palestiniens... op.cit., p. 80-81; Katherine Stewart Howe [dir.], 
pour comprendre les dynamiques plus générales de l'image britannique de la Palestine se justifie à plusieurs titres. Parmi les adeptes du collodion humide qui développent le registre de la photographie de l'Orient dans la seconde moitié du XIX siècle, Frank Mason Good a produit une œuvre dont l'analyse permet de mieux saisir si un regard proprement britannique a pu exister face aux travaux d'autres photographes?. Moins visible dans les archives que ses plus illustres contemporains à cause d'erreurs régulières d'attribution, il a l'avantage aux yeux de l'historien d'être très sensible aux courants esthétiques dominants. Il est en outre un photographe productif et résolument orienté vers la photographie commerciale. Frank Mason, comme le souligne Ken Jacobson, est un bon exemple de ces praticiens itinérants qui parcourent l'Orient dans les années 1860 et $1870^{10}$. Plus artisan qu'artiste aux yeux de ses contemporains, il apparait comme l'un des nombreux instruments du développement de la culture visuelle moyenne qui se construit à partir des années 1860 en Grande-Bretagne.

\section{VARIATIONS PRÉRAPHAÉLITES}

La génération des photographes britanniques partie dans les années 1860 réaliser des prises de vues en Palestine est marquée par un courant esthétique, les préraphaélites, dont l'influence reste limitée sur le continent ${ }^{11}$. Le développement concomitant d'une peinture pour laquelle la Terre Sainte est un sujet de prédilection et d'une première photographie commerciale de l'Orient biblique favorise les interactions entre les deux supports. L'existence de ce mouvement distingue de ce point de vue le regard britannique sur la région. La qualité préraphaélite des prises de vue orientales ou anglaises du photo-

Revealing the Holy Land: the photographic exploration of Palestine, Santa Barbara, Santa Barbara Museum of Art, 1997, p. 122-123 ou Colin Osman, Jerusalem: caught in time, New York, New York University Press, 2000, p. 155.

9. Frank Mason Good est le fils d'un chimiste et pharmacien londonien, Thomas Good. Né en 1839, il semble s'être formé auprès de son père dans le quartier d'Aldersgate. Au début des années 1860, le jeune photographe possède son studio à Londres. Il devient membre de la London Photographic Society en 1864. Il part en Orient de la fin 1866 au printemps 1867 pour le compte de Francis Frith, auquel les prises de vue sont parfois encore attribuées (cf. Bertrand Lazard, "Frank Mason Good and his Middle East Photographs", The Photohistorian, 93 (1991), p. 46-52). Il fait un second voyage à la fin de l'année 1868 et jusqu'au printemps 1869. À son retour, il déménage dans le village d'Hartley Wintney dans le Hampshire où il a un fils et crée son studio. De la fin de l'année 1871 au début de l'année 1872, Good accomplit un parcours assez classique entre Égypte, Constantinople et Malte. Il effectue un dernier voyage en 1875. Après ces quatre voyages, Frank Mason Good dispose en effet d'une banque d'images orientales qu'il exploite pendant des décennies sous une multitude de formats. Il meurt à l'âge de 89 ans dans l'anonymat.

10.Ken Jacobson, Odalisques \& Arabesques: Orientalist Photography 1839-1925, London, Quaritch, 2007 , p. 33.

11. Des travaux récents ont su cependant démontrer que les préraphaélites ne sont pas le courant esthétique totalement isolé longtemps décrit; voir à ce propos Susan P. Casteras et Alicia C. Faxon, PreRaphaelite Art in Its European Context, London, Associated University Presses, 1995. 
graphe Francis Bedford ne fait pas de doute ${ }^{12}$. L'école préraphaélite multiplie les échanges entre peinture et photographie. Le tableau de Thomas Seddon, Jerusalem and the Valley of Jehoshaphat from the Hill of Evil Counsel, peint entre 1854 et 1855, en est un témoignage. L'entreprise de Seddon, qui met près de 120 jours de travail acharné pour réaliser cette œuvre, vise à offrir une vue des lieux du Calvaire qui ne soit pas éloignée de la réalité naturelle de la Palestine ${ }^{13}$. Il s'aide de photographies de James Graham pour compléter son travail une fois revenu en Europe ${ }^{14}$. Cette situation n'est pas complétement inédite en Europe. De tels liens entre peinture de l'Orient et photographie ne sont pas une exception britannique. On sait par exemple comment Eugène Fromentin a recours à un tirage de $\mathrm{W}$. Hammerschmidt pour réaliser son tableau intitulé Sur le Nill5. La spécificité britannique en la matière tient avant tout à l'intensité de ces échanges, dans la rivalité puis l'émulation, entre peinture et photographie.

L'œuvre de Frank Mason Good, avant tout commerciale, témoigne de la diffusion très large des conceptions préraphaélites dans la photographie britannique. On l'observe en particulier dans un tirage intitulé $A$ Carpenter's shop [Fig. 1]. La prise de vue est réalisée par Good durant le dernier de ses quatre voyages en Orient, en 1875. Elle est l'un des exemples les plus clairs de la large diffusion des thématiques chères aux peintres préraphaélites dans la photographie britannique contemporaine. Michael Bartram voit justement dans cette prise de vue un écho d'un des tableaux les plus populaires de Holman Hunt intitulé The Shadow of Death, exposé en 1874 à Londres, avant le départ du photographe en Orient ${ }^{16}$. Par sa capacité à rappeler le Jésus historique et par le réalisme apparent que lui confere une photographie in situ, réalisée à Nazareth même, $A$ Carpenter's shop répond précisément aux injonctions des préraphaélites. L'image respecte une esthétique qui insiste sur la recherche de la validité scientifique, sur la nécessaire similitude entre l'image et la Palestine réelle. Good cherche dans l'enfance de Jésus et dans l'atelier de son père une possibilité de méditation artistique et religieuse fondée sur l'authenticité. Comme dans cet autre tableau préraphaélite fameux qu' est Christ in the House of His Parents de John Everett Millais, c'est à la fois la Passion inéluctable, mais aussi l'enfance et l'humanité de Jésus que la photographie explore ${ }^{17}$.

12. Cf. Diane Waggoner et T. J. Barringer [dir.], The Pre-Raphaelite Lens: British Photography and Painting, 1848-1875, Washington, National Gallery of Art, 2010.

13. Cf. Yehoshua Ben-Arieh, Painting the Holy Land in the nineteenth century, New York, Hemed Books, 1997, p. 130.

14. Allen Staley et Christopher Newall, Pre-Raphaelite Vision: Truth to Nature, London, Tate Publishing, 2004, p. 110.

15. James Thompson et Barbara Wright, La vie et l'cuvre d'Eugène Fromentin, Paris, ACR, 1987, p. 266. 16. William Holman Hunt, The Shadow of Death, 1869-73, huile sur toile, $214.2 \mathrm{~cm} \times 168.2 \mathrm{~cm}$, Manchester City Art Galleries.

17. John E. Millais, Christ in the House of His Parents ('The Carpenter's Shop'), 1849-1850, huile sur toile, 86,4 x $139,7 \mathrm{~cm}$, Tate Gallery, Londres. 


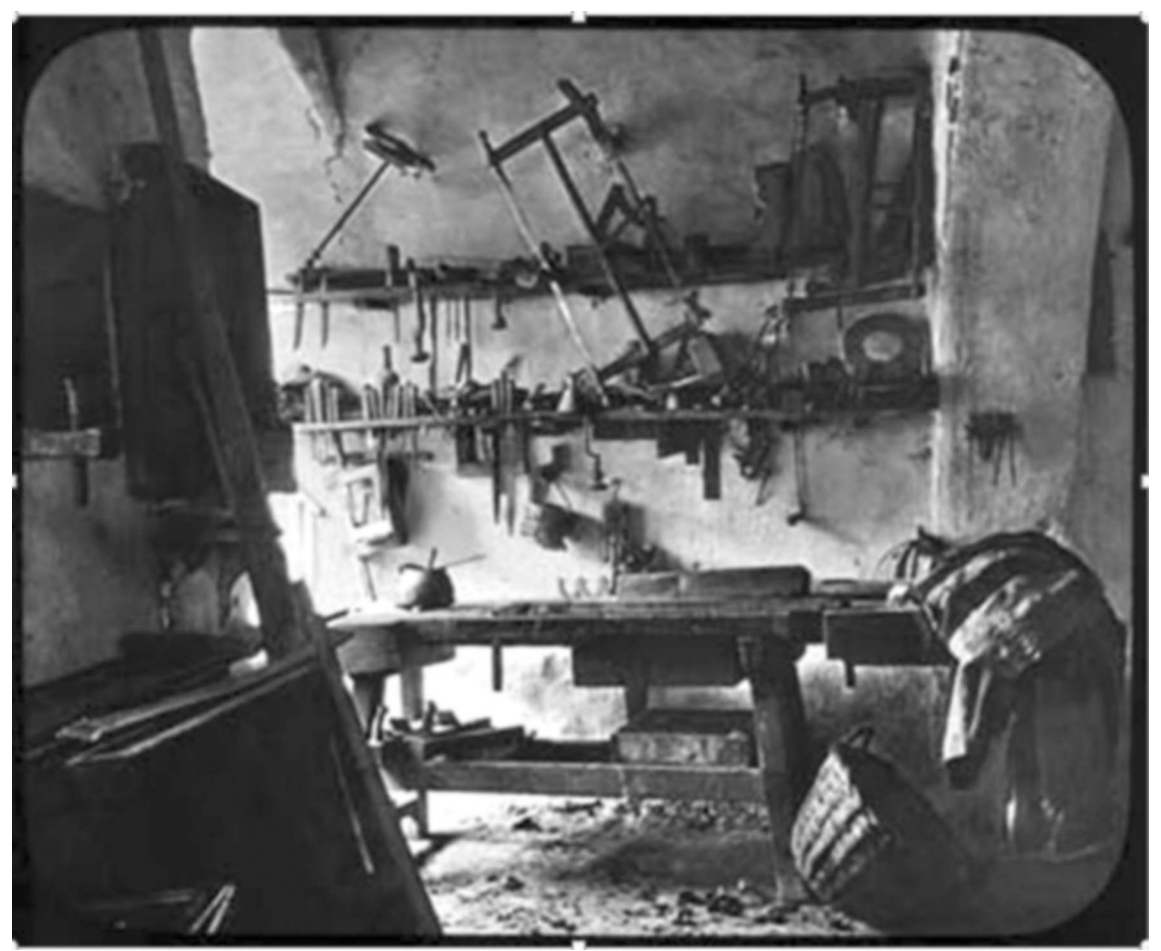

Fig. 1. Frank Mason Good, A Carpenter's Shop, plaque colorisée de lanterne magique (8x8 $\mathrm{cm}$ ), éditée par G.W. Wilson, fin des années 1880 , à partir d'un négatif sur plaque de verre au collodion réalisé en 1875, Presbyterian Church Archives, Nouvelle-Zélande.

Pour mieux attester des liens directs qui existent entre un photographe tel que Frank Mason Good et l'esthétique préraphaélite contemporaine, il faut comprendre dans quel contexte $A$ Carpenter's Shop est réalisé. Même si la photographie de Good se distingue de l'original par sa composition et son point de vue, $A$ Carpenter's shop est une variation élaborée à partir d'une gravure réalisée par Holman Hunt que Frederic Farrar utilise en frontispice de son édition de 1874 de The Life of Christ. Farrar est un proche du peintre, qui réalise ce travail à sa demande ${ }^{18}$. Cette biographie de Jésus, l'un des grands succès de librairie de la seconde moitié du XIX $\mathrm{X}^{\mathrm{e}}$ siècle, connaît des dizaines de rééditions. Ses ventes en Grande-Bretagne ${ }^{19}$ s'élèvent à plus de cent mille exemplaires. Passé son succès initial, l'auteur envisage d'utiliser des photographies susceptibles servir de base à des gravures plus véridiques. Frank Mason Good est appelé par Frederic Farrar à participer à l'illustration de l'édition de

18. Reginald Farrar, The life of Frederic Farrar, London, Nisbet, 1904, p. 95. Farrar écrivit aussi une des premières études biographiques de Holman Hunt: William Holman Hunt, his life and work, London, Virtue, 1893.

19. Boyd Hilton, The Age of atonement: the influence of evangelicalism on social and economic thought, 1795-1865, Oxford, Clarendon Press, 1988, p. 275. 
1876 de The Life of Christ [fig. 2 et 3]. Le dernier voyage du photographe est donc accompli à la demande de Farrar ${ }^{20}$.

Ce dernier ne fait pas le choix de Frank Mason au hasard. Farrar connaît certainement les vues prises par Good dans ses précédents voyages, saluées dans la presse ${ }^{21}$. Il sait aussi que s'inscrire dans la continuité de la représentation préraphaélite de la Terre Sainte n'est pas insurmontable pour F. M. Good. A Carpenter's shop n'est pas une image isolée dans son œuvre. Ses photographies reconnues de paysages anglais montrent déjà l'influence qu'exerça l'esthétique préraphaélite sur ses compositions ${ }^{22}$. Et si l'on ne peut que regretter l'absence de sources écrites qui attesteraient de liens directs entre le photographe et les artistes préraphaélites, les indices concordent pour le placer au moins à la marge de ce cercle, lui qui appartenait à la société de photographie de Londres, longtemps présidée par Francis Bedford, et qui exposait des œuvres aux côtés de celles de Julia Margaret Cameron, autre figure notoire du préraphaélisme en photographie ${ }^{23}$. Une partie des tirages palestiniens de Frank Mason Good sont en outre de véritable copies de vues réalisées en 1862 par Francis Bedford. Mais à la différence de l'aristocratique Bedford, Mason Good, comme son employeur d'un moment Francis Frith, produit une photographie de Palestine à la fois marquée par des Arts plus respectables et de plus en plus largement diffusée. C'est dans cette forme de vulgarisation de l'esthétique préraphaélite que réside l'une des particularités de la représentation de la Palestine par les principaux photographes commerciaux installés en Grande-Bretagne dans les années 1860 et 1870 .

\section{DÉbats THÉOlogiques EN IMAGES}

L'Église anglicane, comme l'Église d'Écosse, est traversée au XIX siècle par de virulents débats entretenus par les questions posées par le dynamisme de l'évangélisme autant que par les avancées de la géologie ou de la biologie.

20. Cf. p. xxiv de l'édition de 1876 .

21. The Photographic News: A Weekly Record of the Progress of Photography, VII, décembre 1863, p. 587 ou The Illustrated Photographer, 20 mars 1869, p. 82.

22. Michael Bartram, The Pre-Raphaelite Camera, op.cit., p. 88-89. Cela est évident dans les photographies de l'île de Wight publiée par James Ware Redding, The Isle of Wight, Illustrated by Photographs, London, 1869, 182 p., ou dans une photographie intitulée Trees after the Storm, vendue le 13 novembre 2001 par Christiés à Los Angeles.

23. Le site Photographic Exhibitions in Britain 1839-1865 (http://peib.dmu.ac.uk) permet de consulter les catalogues de centaines d'expositions photographiques. On retrouve des photographies de Frank Mason Good aux côtés de travaux de Julia Margaret Cameron à l'exposition de la société de photographie d'Edimbourg en 1864. Il est exposé à Londres la même année par la société de photographie de Londres, dont il est membre, en même temps que Francis Bedford, qui est alors vice-président de l'institution, ou Henry Peach Robinson, un photographe très proche du mouvement préraphaélite. 


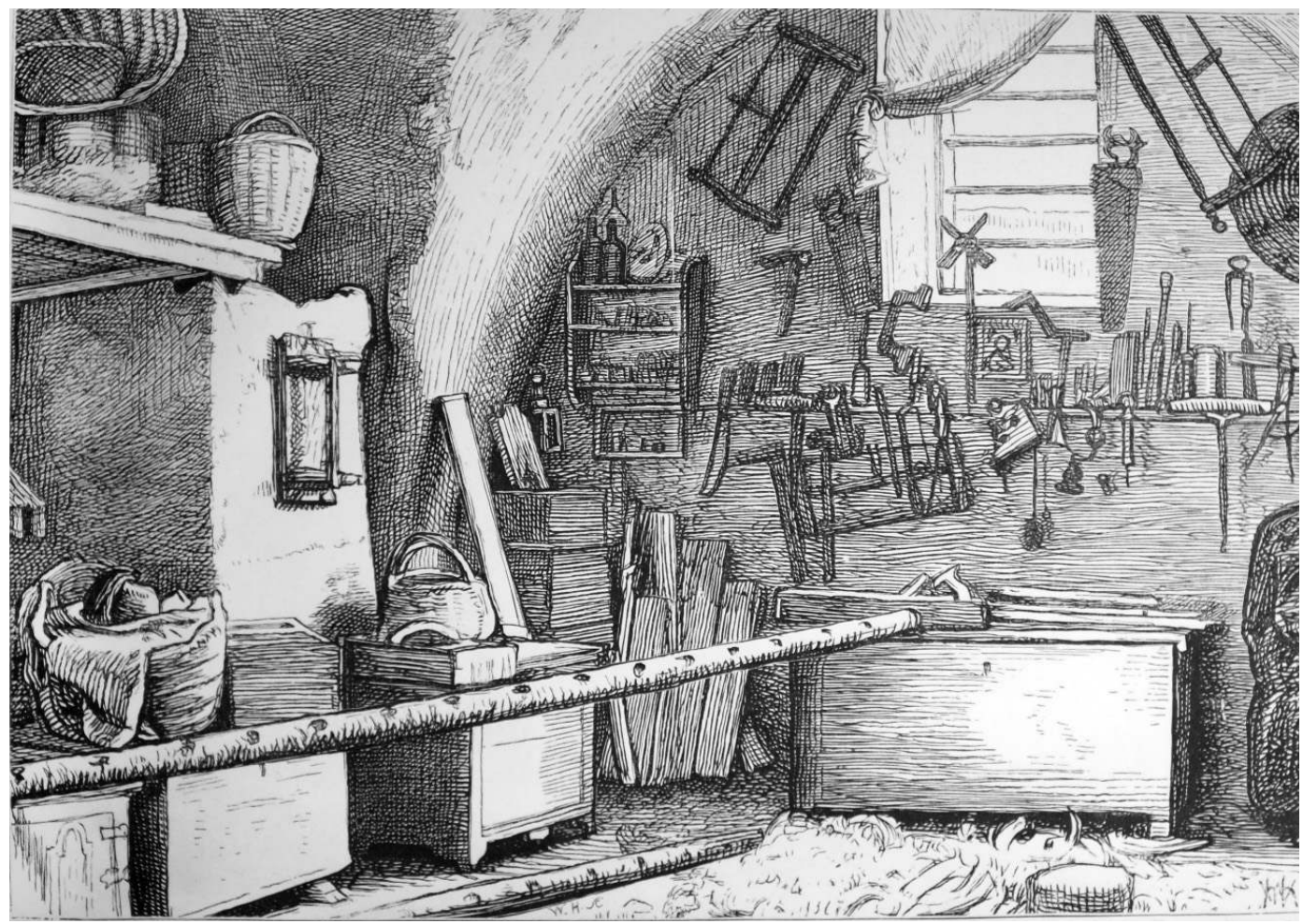

Fig. 2. William Holman Hunt, Interior of a Carpenter's shop at Nazareth, gravure, frontispice de l'édition de 1874 de William Farrar, The Life of Christ, Londres, Cassell, vol I.

Fig. 3. Gravure réalisée à partir d'une photographie de Frank Mason Good pour William Farrar, The Life Of Christ, Londres, Cassell, 1876, p. 50. so

THE LIFE OF CHRIST.

it was regaried, the little town of Nazareth, sitnated as it was in a healthy and secluded valley, yet close upon the confines of great nations, and in the centro of a mixed population, was eminently fitted to be the home of our Saviour's childhood, the scene of that quiet growth " in wisdom, and stature, and faxour with God and man."

Jordan ralley. To get over such flagrant carchessess in the laumting question of the Jewn, worre bare

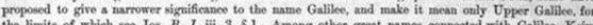
the limits of which see Jox. B. J. iili. 3, \$1. Amogg other great names competed with Gallitee, Keim

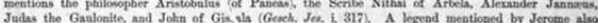
Jades the Gadonite, asd Jokn of Giv. ala (Geech. Jee. i. 317). A kegend mentioned by Jerome also 1.

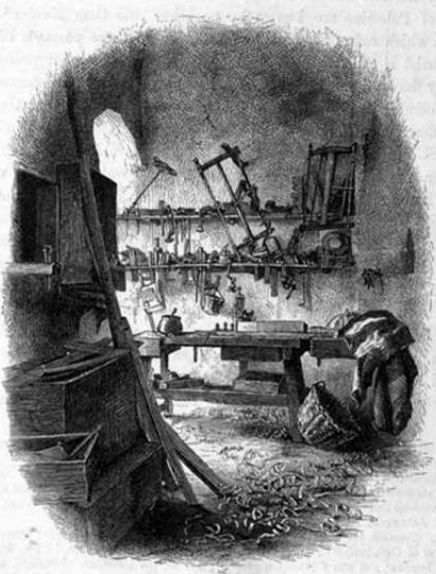

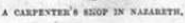


L'imagerie de la Terre Sainte devient l'un des terrains sur lequel s'opposent les théologies ${ }^{24}$.

Dès l'invention du daguerréotype, le procédé photographique est mis à contribution pour défendre une lecture millénariste du paysage palestinien par George Skene Keith, fils d'un éminent membre de la Free Kirk, Alexander Keith, déjà reconnu comme un spécialiste des prophéties ${ }^{25}$. Différentes écoles d'interprétation s'affrontent alors au sein du protestantisme britannique afin d'envisager la fin des temps ${ }^{26}$. Alexander Keith est l'une des figures les plus reconnues de ce millénarisme institutionnalisé, grâce au succès de son ouvrage intitule Evidence of the Truth of the Christian Religion, dont la première publication remonte à $1823^{27}$. Il prend conscience très tôt du fait que la photographie pourrait soutenir son discours. Aussi enrôle-t-il son fils dans un voyage en Palestine en 1844. Les deux hommes prennent plusieurs dizaines de daguerréotypes, aujourd'hui perdus. À leur retour en GrandeBretagne, les Keith publient une nouvelle version de l'ouvrage. Le livre s'enrichit de 18 gravures qui reproduisent les daguerréotypes pris en Palestine. Le livre connaît un succès considérable au fil de ses rééditions jusqu'aux années 1860. Loin d'être isolée, cette création d'une lecture eschatologique de la Palestine par les Keith est concomitante de la reconnaissance publique de la peinture de John Martin. Ses immenses tableaux représentent des catastrophes bibliques dans des formats monumentaux proches du diorama ${ }^{28}$. Cette approche de l'image de la Terre Sainte marquée par un évangélisme millénariste inspire de même les éditions tardives de la Bible illustrée de John Kitto $^{29}$. On retrouve une représentation de la Palestine de plus en plus éloignée des canons de la peinture européenne dans une partie des publications liées à la Low Church anglicane ${ }^{30}$.

\footnotetext{
24. Sur certains éléments du débat, $c f$. Carter Grayson [dir.], Anglican Evangelicals, Protestant Secessions from the Via Media, Oxford, Oxford University Press, 2001.

25. Sur les rapports entre les Eglises écossaises et la Palestine $c f$. Michael Marten, Attempting to bring the gospel home: Scottish missions to Palestine, 1839-1917, London, I.B. Tauris, 2006.

26. J.F.C. Harrison., The second coming: popular millenarianism, 1780-1850, 1979, New Brunswick, Rutgers University Press, 1979.

27.Alexander Keith, Evidence of the Truth of the Christian Religion: Derived from the Literal Fulfillment of Prophecy; Particularly as Illustrated by the History of the Jews, and by the Discoveries of Recent Travellers,

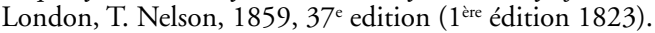

28. Cf. John Martin, The Great Day of his Wrath, huile sur toile, $1851-1853,196,5 \mathrm{~cm}$ x $303,2 \mathrm{~cm}$, Tate Gallery, Londres.

29. John Kitto, The Pictorial Bible, London, Chambers, 1861, vol. 3, p. 261: 'The line of illustration which we propose to take has already been well trodden by Bishop Newton, and, more lately, by Dr. Keith, who between them, have very nearly exhausted the existing stores of information on the subjects of which they treat.'

30. Au XIX' siècle, la Low Church désigne l'ensemble des croyants marqués par les idées évangéliques et le non-conformisme en matière de liturgie et de hiérarchie. À l'opposé, les membres de la High Church défendent l'Église établie dans ses structures traditionnelles et ses rituels, souvent synonyme de conservatisme, l'expression désigne peu à peu les défenseurs de l'anglo-catholicisme, tel John Newman. La Broad Church constitue le versant libéral du protestantisme anglican. Sa théologie est ouverte aux positions contradictoires et à une lecture critique et historique de la Bible.
} 
D'autres visions du paysage et des ruines de Palestine viennent se confronter à ces interprétations apocalyptiques. Le voyage du Prince de Galles en Palestine en 1862 s'inscrit dans ce débat. La présence d'Arthur Penryn Stanley, un homme de la Broad Church, permet d'offrir une lecture des sites bibliques bien différente de celle adoptée par les tenants du millénarisme. Dans les traces qui parsèment la Terre Sainte, Stanley ne voit ni des lieux à conquérir, ni des signes eschatologiques. Son sermon prêché à Jaffa devant le Prince de Galles le 31 mars 1862 le montre: «Il est, grâce à Dieu, parfaitement possible d'être juste et saint et bon sans venir en Palestine. Le pèlerinage n'est pas réellement une obligation pour le chrétien. Les Lieux Saints ne sont pas sacrés au regard de Dieu, seuls les sentiments qu'ils suscitent le sont. Les Croisés étaient dans l'erreur quand ils crurent sauver leur âme en combattant pour reconquérir la Terre Sainte. Ce n'est pas la Jérusalem terrestre, mais la Jérusalem céleste qui est notre "mère à tous », notre mère dans le sens le plus large et le plus engageant $» .31$

Les photographies de Francis Bedford, qui accompagne Stanley dans son voyage, ont pour rôle de montrer ces fragments de la Terre Sainte et de faire naître les sentiments que le prêcheur appelle de ses vœux ${ }^{32}$. Elles servent une lecture particulière de la Palestine qui s'affronte à celles proposées plus tôt par un millénariste tel que Keith. Les préraphaélites jouent leur part dans cette confrontation par l'image. Le souci de l'authenticité d'un Thomas Seddon le met alors en opposition avec les représentations développées par des groupes tels que la Christian Knowledge Society. L'enjeu que représente l'image de la Palestine en Grande-Bretagne au cour du XIX ${ }^{\mathrm{e}}$ siècle ne concerne pas uniquement les productions artistiques les plus élitistes. L'existence d'un tirage de Frank Mason Good, sans équivalent à l'époque, montre que la photographie commerciale de l'Orient, dont il est l'un des acteurs majeurs, n'échappe pas à ce débat en images. Il s'agit de The Summit of the Hill of Evil Counsel [fig. 4]. C'est sur cette colline que se trouvait la maison du prêtre Caïphe selon une tradition médiévale. D’après Luc (XVII, 2), «les prêtres, les scribes et les Pharisiens y tinrent conseil contre Jésus pour le faire mourir ". Judas Iscariote y reçut les trente pièces d'argent qui furent le prix de sa trahison. Une théorie, répandue à partir des années 1860 dans la littérature religieuse britannique, veut que l'arbre imposant qui se trouve alors au sommet de la

31. Arthur Penrhyn Stanley, Sermons preached before His Royal Highness The Prince of Wales during his tour in the East in the Spring of 1862, London, Spottiswoode, p. 27: "It is, thank God, perfectly possible to be just, and holy, and good, without coming to Palestine. Pilgrimage is not really a Christian duty. Holy Places are not really holy in the sight of God, except for the feelings that they produce. The Crusaders were in error, when they thought to save their souls by fighting to regain the Holy Land. It is not the earthly, but the heavenly Jerusalem, which is "the mother of us all" - our mother in the widest and most endearing sense."

32. Francis Bedford, Photographic pictures made by Mr. Francis Bedford during the Tour in the East in which, by command, he accompanied H. R. H. the Prince of Wales, London, Day and Son, 1863, 4 volumes. 


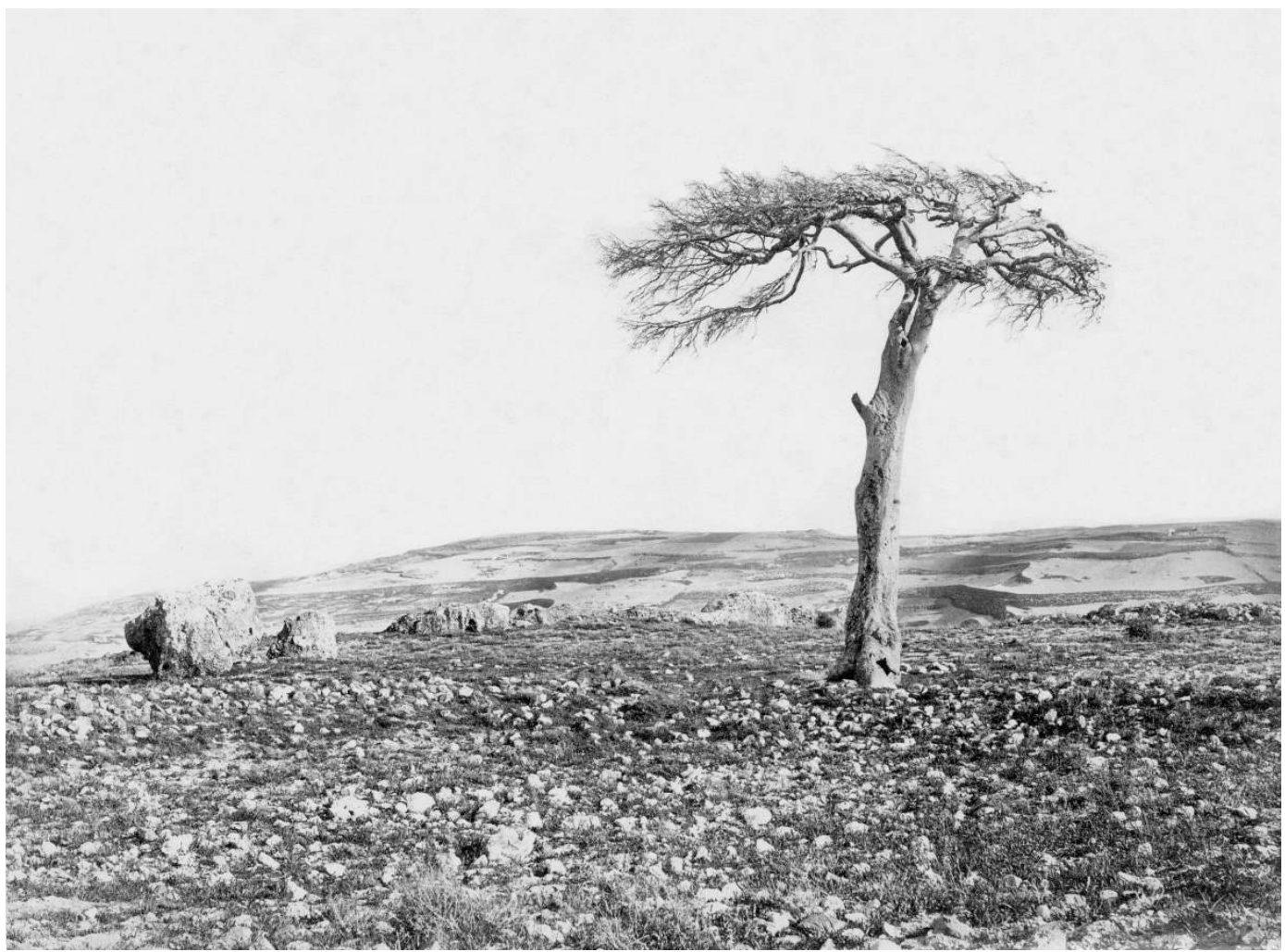

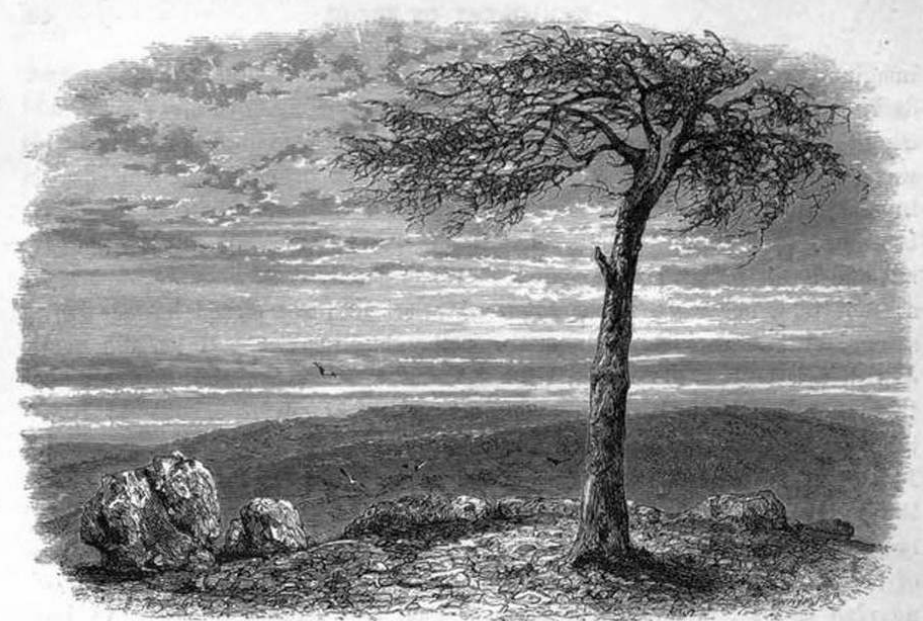

THE HEL OY EVIL COUSSEL.

CHAPTER XLIX.

PALM SENDAY.

"Ride on, ride on in majesty,

In lowly pomp ride on to die !"-Hrus.
Fig. 4. Frank Mason Good, Tree south west of Jerusalem traditionally claimed to be the tree on which Judas Iscariot hanged himself, tirage sur papier albuminé à partir d'un négatif sur plaque de verre au collodion réalisé en 1875 , $\mathrm{n}^{\circ} 1312$ dans le catalogue Mansell, avec la permission du Palestine Exploration Fund (Londres).

Fig. 5. William Farrar, The Life Of Christ, London, Cassell, 1876 (15e edition), p. 530. 
colline fut précisément celui qui servit au suicide par pendaison de Judas ${ }^{33}$. La photographie de Good participe de cet effort de réalisme, véritable arme dans les débats qui opposent différentes cultures visuelles de la Palestine en Grande-Bretagne. Elle s'inscrit dans la lignée des réflexions de Thomas de Quincey. Dans son Judas Iscariot, il tente de dresser le portrait d'un apôtre nécessaire à la Passion, un révolutionnaire convaincu de l'imminence du Royaume dont il aurait cherché, par sa trahison, à hâter la venue. Farrar n'hésite pas à considérer de Quincey comme une référence et à faire figurer une gravure inspirée de l'image de Good dans sa biographie de Jésus [fig. 5]. Le regard de Frank Mason Good est typique d'un homme de la Broad Church. Le peu de documentation qui subsiste sur le photographe confirme cette conclusion de manière indirecte. Une photographie de Charles Kingsley par F.M. Good prise au début des années 1870 suggère une proximité entre le photographe et son sujet ${ }^{34}$. Charles Kingsley, connu avant tout pour ses romans, était, comme Stanley, un homme de la Broad Church, sensible aux questions liées aux réformes sociales ${ }^{35}$. Les liens entre Good et Farrar, lui aussi proche du groupe d'intellectuels latitudinaires qui constitue la tête pensante de la Broad Church, renforcent encore l'hypothèse selon laquelle le photographe fut l'un des acteurs de l'élaboration visuelle de la Terre Sainte du point de vue de l'Anglicanisme libéral.

L'intensité des débats théologiques constitue l'un des éléments qui distinguent la photographe orientaliste britannique de la seconde moitié du $\mathrm{XIX}^{\mathrm{e}}$ siècle. Good n'en est qu'un des nombreux acteurs.

\section{UNE GÉOGRAPHIE PROTESTANTE?}

Comme en Égypte, où les étapes du parcours photographique sont très tôt fixées, l'imagerie européenne de la Palestine semble figée dans le ressassement des mêmes thèmes, voire des mêmes compositions ${ }^{36}$. L'indigène n'est généralement dans l'image que pour donner un sens des proportions ou pour servir de témoin de l'aspect supposément immuable de la vie en Terre Sainte. Les photographes britanniques n'échappent pas à cette règle. Ces pèlerinages photographiques que sont souvent les séries de plaques de lanterne magique ou de vues stéréoscopiques réalisées par Francis Frith, ne peuvent ignorer les lieux saints traditionnels au risque de manquer une partie de leur clientèle potentielle. La répétition est parfois telle qu'il est difficile de distinguer

33. Cf. The Sunday at Home, VIII (1861), p. 331, par exemple.

34. Frank Mason Good, portrait de Charles Kingsley, circa 1870, National Portrait Gallery, cote NPG $\mathrm{x} 11879$.

35. Cf. Tod E. Jones, The Broad Church: A Biography of a Movement, Lanham (Maryland), Lexington Books, 2003.

36. Caroline Lehni, Lire l'Égypte en Images, La représentation de l'Égypte au Royaume-Uni à travers lìllustration des récits de voyage (1798-1914), thèse d'études anglophones sous la direction d'Alexis Tadié. 
les travaux des principaux photographes de la région tant certaines de leurs prises de vue semblent être la répétition inlassable de la même image.

Pourtant, si l'on prend ici encore l'exemple du travail de Frank Mason Good, on voit régulièrement surgir des écarts, des images qui échappent aux parcours et aux lieux traditionnels, majoritairement fixés par les traditions orthodoxe et catholique. Dans sa monumentale série de 368 vues stéréoscopiques d'Orient intitulée Eastern Series, l'église de la Nativité ou celle du Saint Sépulcre sont moins représentées que la vallée de Josaphat ou que Béthanie ${ }^{37}$. Ce dernier lieu semble avoir particulièrement intéressé Frank Mason Good, qui en réalise de nombreuses prises de vue, certaines originales, d'autres directement copiées sur les travaux de Francis Frith ${ }^{38}$ [fig. 6]. Sans doute doit-on y voir l'intérêt tout particulier d'un anglican de la Broad Church pour ce lieu dont l'authenticité supposée est alors si évocatrice des derniers jours de Jésus. Béthanie tient une place toute particulière dans la géo-piété britannique. Pour le protestantisme britannique, le village est le lieu de la dernière bénédiction de Jésus, et non pas le sommet du Mont des Oliviers d'où l'église de l'Ascension signale aux yeux des traditions romaines et orthodoxes le lieu des derniers pas de Jésus ressuscité sur Terre ${ }^{39}$. Ce bâtiment n'apparaît d'ailleurs quasiment pas dans la photographie britannique, à l'exception des vues générales du mont des Oliviers ${ }^{40}$. Les tirages individuels sur papier albuminé figurent aussi des lieux souvent délaissés par les traditions continentales, tel Shiloh ou Samarie. Frank Mason Good n'est évidemment pas le seul acteur de cette recomposition de la géographie sainte propre au protestantisme britannique. Comme Francis Frith dont il s'inspire, il est l'un des acteurs d'une construction du paysage palestinien qui se manifeste dans la plupart des ouvrages britanniques consacrés alors au sujet.

Ceci est notamment visible dans un ouvrage d'Henry Baker Tristram, Scenes in the East, publié pour le compte de la société anglicane pour la promotion du savoir chrétien ${ }^{41}$. Le lecteur est invité au voyage en Palestine mais les lieux saints les plus importants des traditions orthodoxe et catholique sont soigneusement évités, dans une démarche qui apparaît dès l'époque comme une façon de se démarquer de l'illustration traditionnelle de la Terre

37. Un catalogue des vues stéréoscopiques de Frank Mason Good est régulièrement mise à jour par la National Stereoscopic Association (Etats-Unis) : The Stereoviews of Frank Mason Good, The Institute for Photographic Research, View List series $n^{\circ} 7,2000$.

38. La vue stéréoscopique $\mathrm{n}^{\circ} 21$ a des Eastern Series (1721 dans la série Mansell) est ainsi la copie conforme d'une vue réalisée en 1857 par Frith (n² 422 de la série de vues stéréoscopiques intitulée Views in the Holy Land et publiée entre 1857 et 1859).

39. Cf. A. L. Harkness, Sketch of a tour in the Holy Land, London, Partridge, 1869, p. 32.

40. C'est le cas d'une vue générale réalisée en 1857 par Francis Frith (Francis Frith, Sinai, Palestine, The Nile, London, Mackenzie, $\left.\mathrm{n}^{\circ} 193,1863\right)$, rien de comparable avec la vue de Félix Bonfils intitulée "Coupole de l'Ascension" ( $\mathrm{n}^{\circ} 293$ ), par exemple (un exemplaire est conservé à la Librairie du Congrès américain à Washington, cote LOT 13550, no. 226 [P\&P])

41. Henry Baker Tristram, Scenes in the East: consisting of twelve coloured photographic views of places mentioned in the Bible, with descriptive letter-press, London, Society for Promoting Christian Knowledge, 1870. 


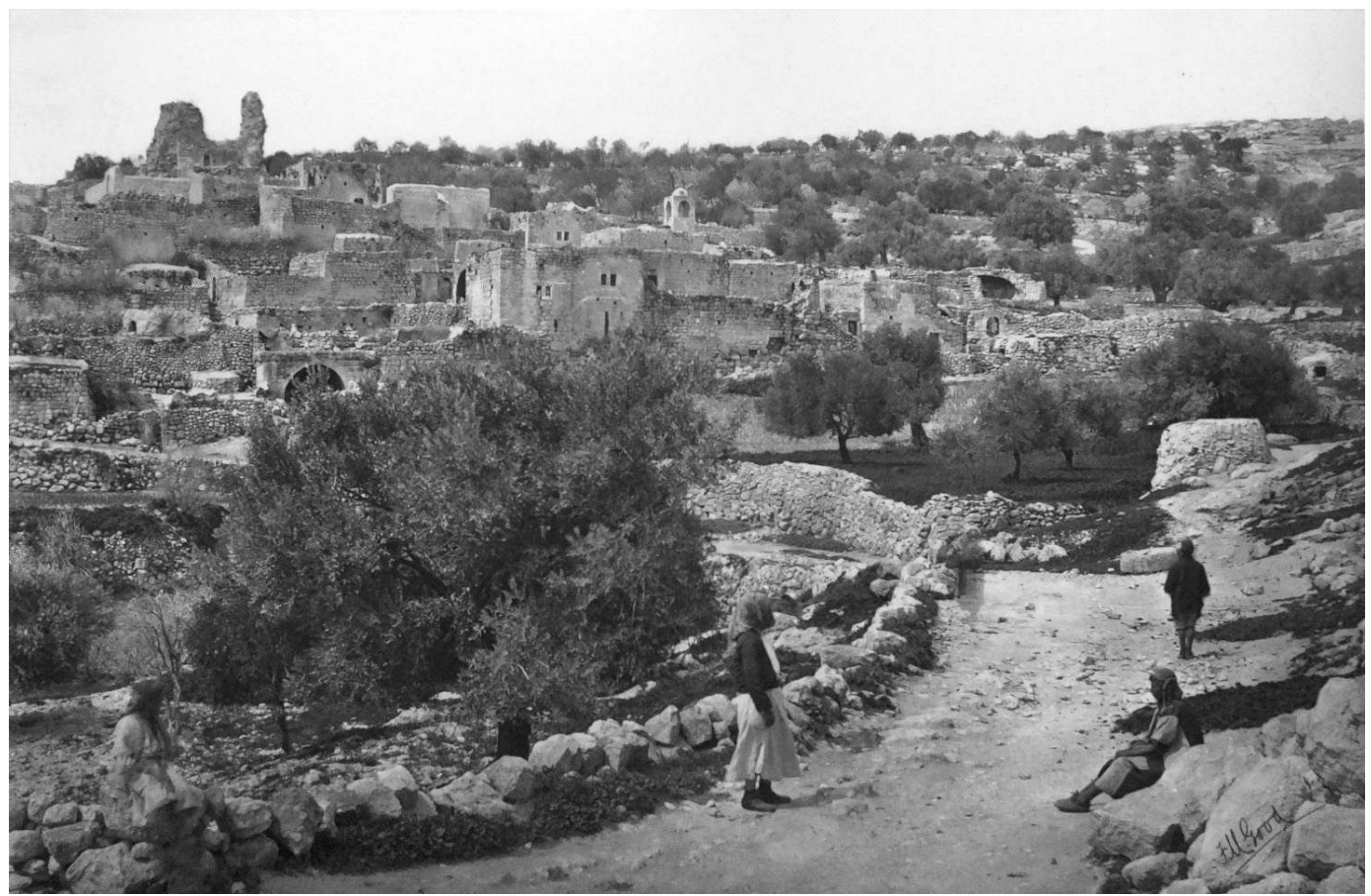

Figure 6. Frank Mason Good, Bethany, tirage sur papier albuminé collé sur carton et relié, in Henry Baker Tristram, Pathways of Palestine, London, S. Low, 1881, volume 1, p. 108, à partir d'un négatif sur plaque de verre au collodion.

Sainte: «Nous ignorons si un quelconque principe de sélection a présidé à cette publication, mais nous ne pouvons recommander ces gravures aux Catholiques sans remarquer que les sites les plus sacrés en sont exclus. Même Béthanie est une maigre compensation pour le Mont des Oliviers, et il n'y a ni vue de Jérusalem elle-même, ni vue du Saint Sépulcre " ${ }^{42}$.

Ce processus est déjà à l'œuvre parmi les membres de la mission envoyée en Palestine par l'Église d'Écosse en 1839. Celle-ci pose un regard érudit sur l'authenticité d'un certain nombre de localisations et n'hésite pas à mettre en doute la validité du positionnement du Saint Sépulcre : «Nous visitâmes l'église du Saint-Sépulcre qu'on regarde généralement comme couvrant le lieu même du calvaire, sur lequel notre Seigneur fut crucifié et enterré. Cette visite n'excita en nous que des sentiments pénibles [...]. Il n'existe aucune tradition qui désigne quelque autre endroit comme le lieu du calvaire, et il

42. The Month, New Series, I (janvier-juillet 1870), p. 376: "We do not know whether any special principle of distinction has presided over the publication, but we cannot commend the engravings themselves to the Catholics without remarking on the exclusion of the most sacred sites. Even Bethany is a poor substitute for the Mount of Olives, and there is no view here either of Jerusalem itself or the Holy Sepulchre." 
nous vint à la pensée qu'un aurait dû le chercher parmi les tombeaux qui couvrent la hauteur qui domine l'étang de Gihon ${ }^{43}$.

Plus tard, le général Gordon, lors de son séjour en Palestine au milieu des années 1880 , indique la localisation de ce qu'il pense être le vrai tombeau de Jésus, à l'extérieur de l'enceinte de Jérusalem. Ce tombeau, situé près de la porte de Naplouse, est encore considéré comme authentique de nos jours par certains voyageurs protestants. Henry Baker Tristram ou Frank Mason Good s'inscrivent en cela dans un mouvement plus large et plus ancien, qui détermine une partie du regard britannique sur la Terre Sainte. Au-delà des clivages théologiques, les protestants britanniques recomposent par la photographie et l'illustration une géographie sainte aussi éloignée que possible de la tradition romaine.

\section{Documenter la Palestine : Ruines et photographie}

Les usages archéologiques et topographiques de la photographie en Palestine ne sont pas exclusivement britanniques. Le calotypiste français Auguste Salzmann part en 1853 en Terre Sainte dans le cadre d'une mission en partie sanctionnée par le ministère de l'Instruction publique. Il veut vérifier les hypothèses polémiques de Félix de Saulcy développées dans Voyage autour de la mer Morte. Son utilisation documentaire de la photographie pour représenter la Terre Sainte est d'une grande nouveautée ${ }^{44}$. Quelques années plus tard, Ermete Pierotti, un officier sarde embauché par le gouverneur ottoman de Jérusalem, publie La Jérusalem explorée, une nouvelle tentative d'étudier la ville avec l'appui de la photographie. L'ouvrage devient rapidement polémique en Grande-Bretagne. Pierotti dédicace son livre à Napoléon III et laisse supposer qu'il dispose d'un soutien français. Il tend par ailleurs à confirmer les identifications les plus traditionnelles des lieux saints, tout en laissant croire que les lithographies qui viennent consolider ses analyses sont réalisées à partir de ses propres photographies, alors qu'il apparaît que le véritable auteur des prises de vues est un photographe résident nommé Mendel Diness ${ }^{45}$. Le livre déclenche une controverse. C'est aux protestants britanniques que revient le droit et la charge de l'étude archéologique et topographique de la Terre Sainte ${ }^{46}$. Les travaux de Charles-Jean-Melchior de Vogüé, qui publie en 1864 un album de photographies de l'esplanade du Temple de Jerusalem, et l'expédition du Duc de Luynes, qui s'adjoint les services de Louis Vignes, officier de marine et pho-

43. Andrew Bonar, Les Juifs d'Europe et de Palestine, traduction Louis Gaussen, Paris, Delay, 1844, p. 114-116.

44. Stéphane Michaud, Jean-Yves Mollier et Nicole Savy [dir.], Usages de l'image au XIX'e siècle, Paris, Créaphis, 1992, p.131-132

45. Hisham Khatib, Palestine and Egypt Under the Ottomans: Paintings, Books, Photographs, Maps, p. 233. 46. James Moscrop, Measuring Jerusalem: the Palestine Exploration Fund and British interests in the Holy Land, London, Leicester University Press, 2000, p. 64 
tographe, afin d'étudier la mer Morte, confirment l'impression que l'investissement français en Terre Sainte va croissant ${ }^{47}$. La naissance de la première entreprise photographique systématique de Jérusalem est de fait une réponse à ce qui est alors perçu comme une outrecuidance française. Certains spécialistes britanniques des études bibliques, tel George Grove, poussent en effet à la création d'un fonds susceptible de financer une étude systématique de la Terre Sainte afin de reprendre la main dans ce domaine. Ce projet prend d'abord la forme du Jerusalem Water Relief Fund en 1864, puis du Palestine Exploration Fund en 1865. À la fois instrument de renseignement militaire, projet de topographie générale de la région, exploration toponymique, archéologique et photographique de la Palestine, ce dernier fonds est très actif dans la production des savoirs sur la Terre Sainte en Europe dans les années 1860 et 1870.

La photographie est immédiatement intégrée aux activités des deux fonds ${ }^{48}$. En juin 1864, lors de la première expédition consacrée à Jérusalem, puis en 1868, lors de l'expédition qui vise à cartographier le Sinaï, James McDonald, un sergent formé à l'école des ingénieurs royaux de Chatham, est chargé des prises de vue. Pour chaque exploration, McDonald réalise des dizaines de photographies selon les préceptes développés à Chatham par Charles Thuston Thompson puis par le capitaine Henry Schaw ${ }^{49}$. Le rôle de Charles William Wilson, lui aussi partie prenante des levers en Palestine, pionnier de l'utilisation de la photographie par les topographes, est aussi à souligner ${ }^{50}$. La photographie de James McDonald differe, par sa codification et son institutionnalisation, de la pratique des photographes voyageurs qui le précèdent. Cette photographie documentaire n'a, au départ, pas de vocation marchande. Elle ne répond pas aux mêmes contraintes que la photographie commerciale de l'époque comme le montre sa moindre propension à l'exotisme ${ }^{51}$. Elle est pourtant diffusée bien audelà du cercle des topographes et des spécialistes du renseignement. Soixantedix-sept de ses prises de vues viennent illustrer la publication des résultats des travaux de 1864, plus d'une centaine sont reproduites dans les trois volumes qui présentent l'exploration du Sinaï ${ }^{52}$. Une partie de ces images est en outre vendue à l'unité en Angleterre.

47. Melchior Vogüé, Temple de Jérusalem, monographie du Haram-ech-Chérif, suivie d'un Essai sur la topographie de la Ville-sainte, Paris, Noblet \& Baudry, 1864, 142 p. et Honoré d'Albert Luynes, Voyage d'exploration à la mer Morte, à Petra, et sur la rive gauche du Jourdain, Paris, A. Bertrand, 1874, 3 volumes. 48. Kathleen Stewart Howe, 'Mapping a Sacred Geography: Photographic Surveys by the Royal Engineers in the Holy Land, 1864-1868, in J.M. Schwartz et J.R. Ryan [dir.], Picturing place: photography and the geographical imagination, London, I.B. Tauris, 2003, p. 195-225

49. John Falconer, 'Photography and the Royal Engineers', The Photographic Collector, II, 3 (1981), p. 33-64.

50. Charles Wilson participe à la commission de délimitation frontalière envoyée en 1859 en Colombie britannique. Il y pratique la photographie à des fins topographiques (voir Andrew Birrell, 'Survey Photography in British Columbia', BC Studies, LII (hiver 1981-82), p. 39-60.

51. Les prises de vue de McDonald publiée dams Ordnance Survey of Jerusalem insistent avant tout sur l'architecture (voir Yeshayahu Nir, The Bible and the image... op.cit., p. 92-93).

52. Charles William Wilson, Henry Spencer Palmer et Henry James [dir.], Ordnance Survey of Mount Sinai, Southampton, Ordnance survey Office, 1869, 5 volumes. 
Tout porte à croire que les travaux de James McDonald ne sont pas ignorés des autres photographes britanniques partis en Palestine et dans le Sinaï. Lœuvre de Frank Mason Good en est, là encore, l'une des traces. En témoignent par exemple ses photographies du Sinaï réalisées durant ses derniers voyages. Les travaux du Palestine Exploration Fund et les photographies de James McDonald contribuent alors à renouveler la liste des hauts lieux de l'Orient biblique. Au Wadi-al-Mukatteb, lieu du miracle de la manne, et au Djebel Serbal, possible mont Sinaï pour les protestants britanniques, déjà photographiés par Francis Frith et Frank Mason durant son premier voyage, on ajoute alors d'autres sites évoqués dans l'Exode ${ }^{53}$. Parmi ceux-ci, on trouve notamment la fontaine de Moïse, proche du Golfe de Suez. Le prophète y aurait fait jaillir l'eau après le passage de la Mer Rouge. Cet endroit reste longtemps secondaire dans la littérature anglophone consacrée à la région. Le très influent Edward Robinson le signale rapidement dans Biblical researches in Palestine, mais le lieu n'apparaît dans aucune des grandes séries photographiques et stéréoscopiques des années 1850 et $1860^{54}$. Il faut attendre les années 1870 pour que la fontaine de Moïse intègre la liste relativement figée des sites à voir en Orient. Une photographie du lieu occupe le frontispice d'un des ouvrages publiés par les explorateurs du Palestine Exploration Fund ${ }^{55}$. Frank Mason Good en propose alors une photographie publiée dans Pathways of Palestine d'Henry Tristram Baker [fig. 7]. Le point de vue est très différent de celui adopté par James McDonald, mais l'apparition du lieu dans la banque d'image d'un photographe commercial n'est pas anodine. Les acteurs du Palestine Exploration Fund mettent en valeur de nouveaux lieux et Good s'adapte à une demande et à des représentations changeantes de l'Orient biblique. Cette relation entre photographie vulgarisatrice et photographie spécialisée n'est pas à sens unique. La lecture de Picturesque Palestine, publié sous les auspices du Palestine Exploration Fund par Charles Wilson, permet de reconnaitre plusieurs illustrations directement réalisées à partir de tirages de Frank Mason Good. Une gravure qui montre Shilo, où fut élevé le Tabernacle, est par exemple une copie d'un tirage réalisé par Good pour Mansell [fig. 8 et 9 9 ${ }^{56}$. Henry Baker Tristram, très impliqué dans les activités du Palestine Exploration Fund utilise lui aussi des photographies de Good dans ses publications ${ }^{57}$. Au-delà du cas particulier de Good, on observe ici les effets du biblicisme qui caractérise l'époque victorienne [fig. 10 ${ }^{58}$. La

\footnotetext{
53. Nickel Douglas, Francis Frith in Egypt and Palestine... op.cit., p. 64.

54. Edward Robinson, Biblical researches in Palestine, Mount Sinai and Arabia Petraea: a journal of travels in the year 1838, London, Murray, p. 62-63.

55. Il s'agit d'une reproduction intitulée Ayun Musa, (Well of Moses) qui figure sur le frontispice de la deuxième partie du volume 3 (Charles William Wilson, Henry Spencer Palmer et Henry James [dir.], Ordnance Survey of Mount Sinai... op.cit.)

56. Charles Wilson, Picturesque Palestine, London, G. Virtue, volume 1, p. 225. Cf. figures 8 et 9.

57. Henry Baker Tristram, Pathways of Palestine... op.cit.

58. La proportion des vues stéréoscopiques consacrées à la Palestine dans les Eastern Series de Good est à ce titre éloquente ( $c f$. figure 10). Plus du tiers des vues de la série, censée couvrir tout l'Orient, sont consacrées à ce territoire.
} 


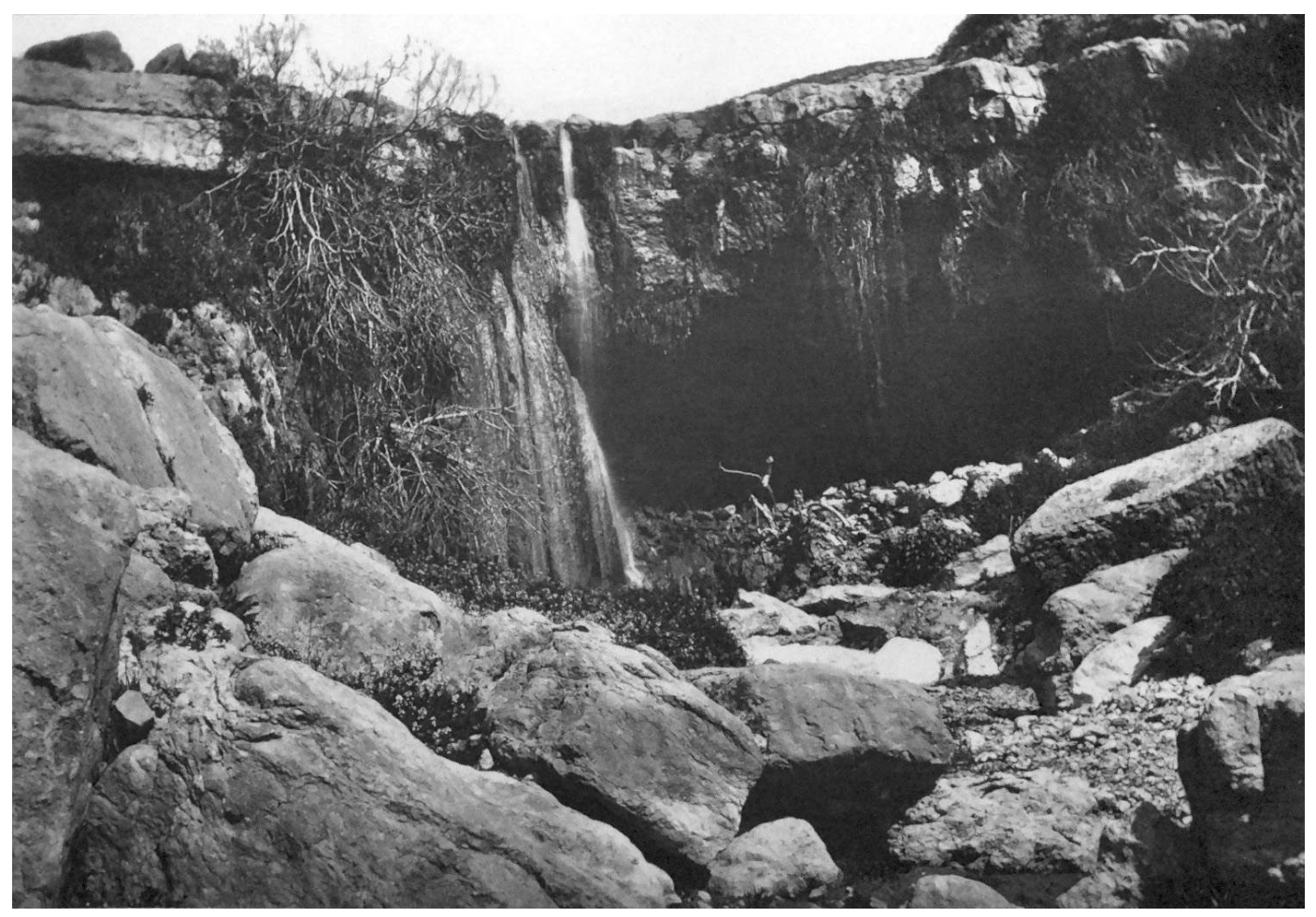

Fig. 7. Frank Mason Good, Ayun Mûsa, under Mount Nebo, tirage sur papier albuminé collé sur carton et relié, Tristram Henry Baker, Pathways of Palestine, London, S. Low, 1881, volume 1, p. 126, à partir d'un négatif sur plaque de verre au collodion.

connaissance approfondie de la Terre Sainte n'est pas réservée aux savants. La photographie britannique de la Palestine en est l'écho. Elle intègre très rapidement les nouvelles découvertes dans sa production commerciale.

\section{IMAGES BRITANNIQUES, DIFFUSION INTERNATIONALE}

Durant les années 1860 et 1870 en particulier, la photographie britannique de la Palestine se distingue des autres productions européennes par ses liens avec le mouvement préraphaélite et son utilisation dans des débats religieux spécifiques. L'exemple de Frank Mason Good démontre toutefois que ces particularismes n'empêchent en rien les usages de ces images bien au-delà de l'Angleterre, du pays de Galles ou de l'Écosse. Le photographe utilise ses centaines de négatifs de l'Orient comme une banque d'images disponibles pour des clients aussi bien britanniques que français ou américains. En témoigne par exemple la publication de certaines de ses photographies 
all Christian villages. In the Middle Ages nearly all this district belonged to the Church, twenty-one villages north of Jerusalem having been given by Godfrey to the Canons of the Holy Sepulchre, while Sinjil, Bethel, Turmus 'Ayya, Mezr'ah, Kefr Malik, and others, were acquired later by this powerful body of ecclesiastics

Traces of the Crusaders are found in all parts of the district, from the two castles which dominate the northern pass, to the rocky fortress of Neby Samwil on the south; at Bireh, Beitin, Jufna, Taiyibeh, Neby Samwil, and Mukatir, ancient churches and chapels remain in ruins; and at Arnutieh and other places there are small forts apparently of Crusading origin.

The narrow pass which we now approach is one of the wildest parts of the road between Nablus and Jerusalem, which here becomes a mere lane thickly strewn with loose stones.

Figure 8. Anon., Seilûn, on the site of Shiloh, gravure d'après une photographie de Frank Mason Good in Charles Wilson,

Picturesque Palestine... op.cit., p. 225.

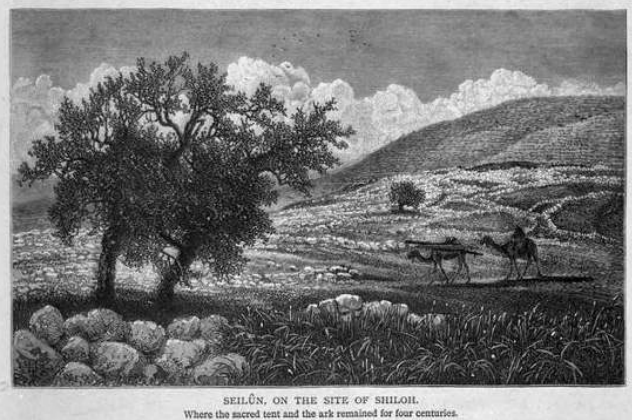

To the left of the road a square building of large drafted stones seems probably to have been a Crusading fort or hostel, and a vertical cliff of hard rock reaches up some twenty or thirty feet, while the steep hill above is terraced and planted with olives. A small spring drips out of the procipice, and is called 'Ain-el-Haramlyeh, "the Robbers' Spring." On the flat rocky bed of the valley a little coarse grass grows near the water, and a group of camels, tawny and dusty, may probably be found reposing in the shade, while their drivers, kneeling on the carefully spread abbas, are reciting the afternoon prayer-a ceremony no doubt religiously observed by the brigands from the nearest village, who once haunted the gorge and gave its mame to the spring. To the right of the road an extremely steep mountainside, terraced in places, and in others belted with low cliffs, dominates the pass, rising more vol. I.

dans Le pays des croisades de Jules Hoche ${ }^{59}$ et la Syrie d'aujourd'hui de Louis Lotret $^{60}$. À Paris, la maison Martinet et le studio Léon Levy commercialisent des tirages de Good $^{61}$. On trouve aussi une version française des séries de plaques de lanterne magique produites par la firme londonienne York and Son. Il existe ainsi une version française des «lectures» vendues par la firme York and Son pour accompagner les vues de lanterne magique ${ }^{62}$. Des clients du continent commandent aussi des tirages à Good à leur retour d'un voyage en Orient, comme le montre l'album de Tony Dutreux conservé à la Bibliothèque Nationale du Luxembourg. Aux États-Unis, un éditeur américain de vues pour lanterne magique commença à publier des plaques réalisées à partir des vues de Good après son dernier voyage. Il s'agit de la firme

59. Jules Hoche, Le Pays des Croisades, Paris, Libraire illustrée, 1886.

60. Louis Lotret, La Syrie d'aujourd'hui, Paris, Hachette, 1884.

61. C'est ce que montre une photographie conservée à la bibliothèque nationale de France (cote: EO658 -BOITE FOL B). La mention et le cachet de ces deux studios apparaissent au dos des tirages albuminés.

62. Anon., Enseignement par les projections lumineuses. Lectures traduites de l'anglais pour accompagner les collections de photographies sur verre de York and Son, Paris, A. Molteni, 1893, tome 9, L'Égypte et tome 10, Voyage en Terre Sainte. 


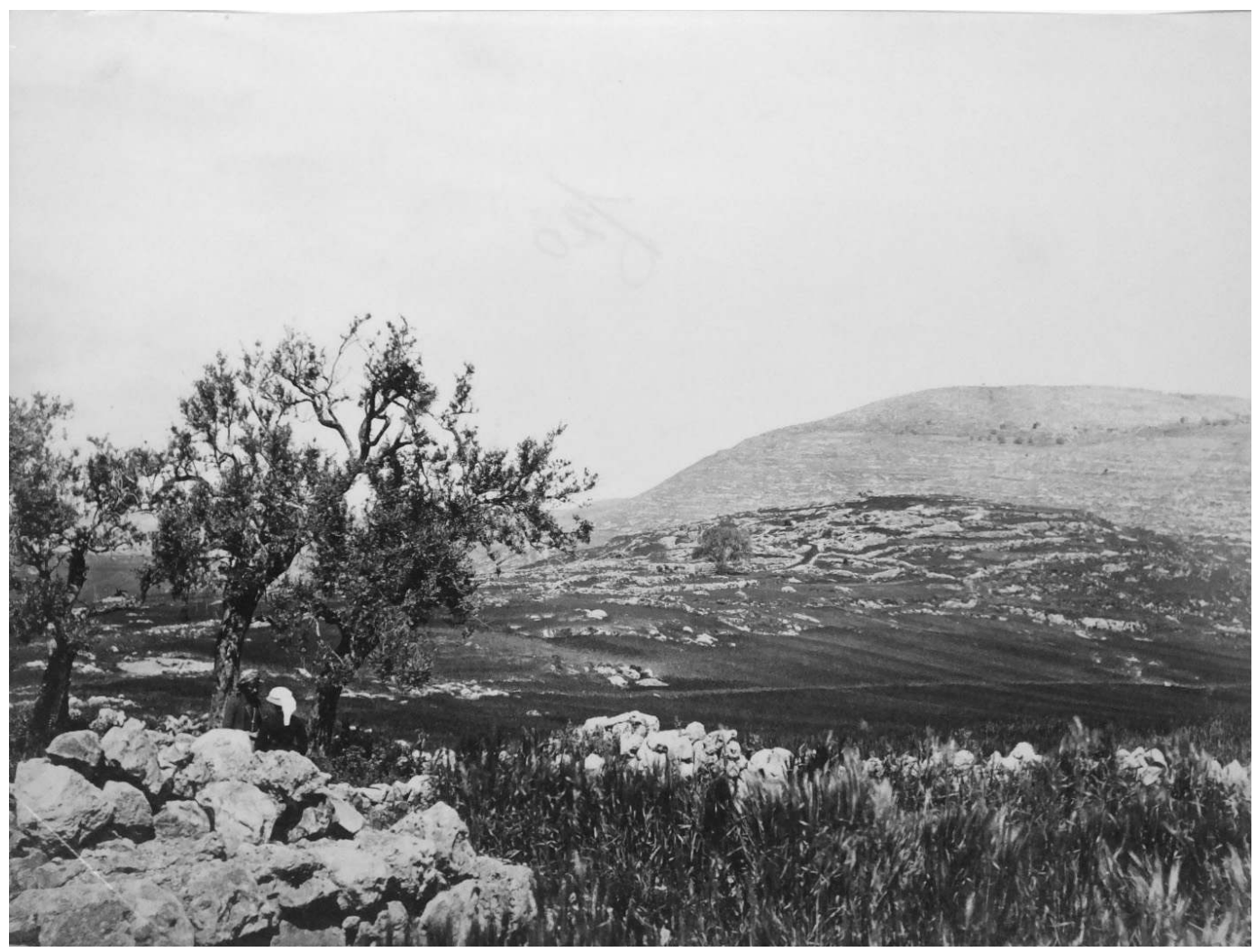

Figure 9. Frank Mason Good, Shiloh, tirage sur papier albuminé édité par Francis Frith (Photo-pictures $n^{\circ} 589$ ) à partir d'un négatif sur plaque de verre au collodion réalisé à la fin des années 1860 .

Figure 10. Proportions des différents espaces représentés dans les Eastern Series de Frank Mason Good (la délimitation retenue pour la Palestine correspond à celle couramment en usage au XIX siècle, «de Dan à Beer-Sheva»).

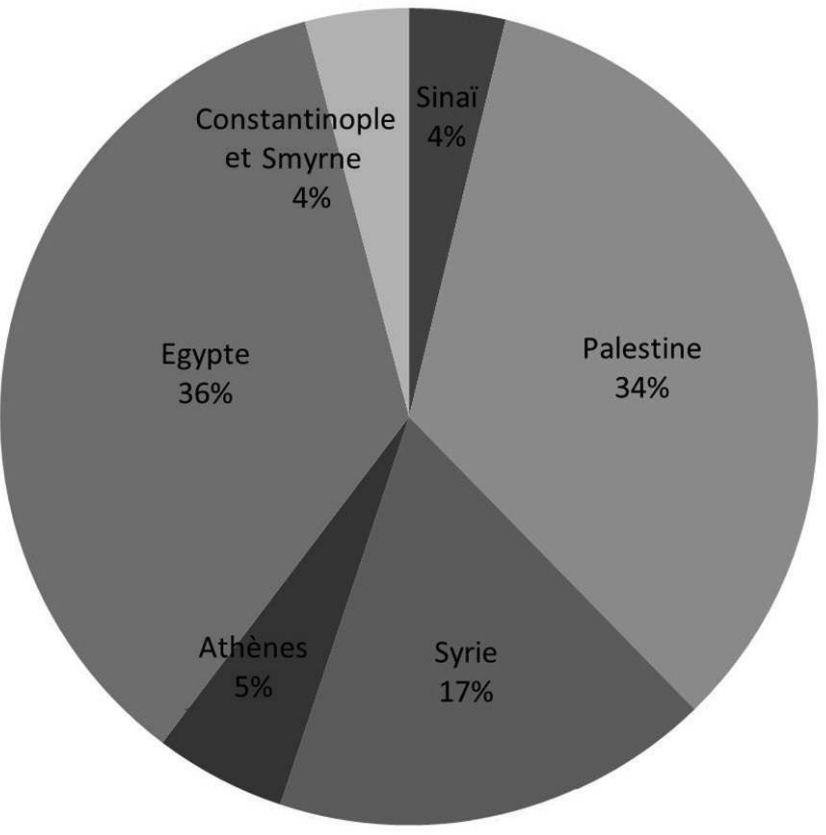


américaine Sciopticon Company, dirigée par Lorenzo Marcy. On a le plus grand mal à dater très précisément les séries mais il est plus probable qu'elles aient été commercialisées à partir de la fin des années 1870 et du début des années 1880. Pour la marque Sciopticon, on possède deux catalogues qui proposent à la vente des plaques produites par Good en 1877 puis en 1888 pour le marché américain ${ }^{63}$. La photographie de Good est aussi exploitée en Allemagne. Ken Jacobson signale ainsi qu'une gravure publiée dans un ouvrage du romancier et égyptologue allemand Georg Ebers, réalisé par B. Strassberger, est directement inspirée d'une prise de vue de Frank Mason Good $^{64}$. Un examen attentif de l'ouvrage, traduit en anglais et publié à Londres en 1880, montre d'ailleurs que plusieurs autres illustrations sont réalisées à partir du travail du photographe britannique ${ }^{65}$.

Frank Mason Good voit ses prises de vues diffusées de manière illégale à grande échelle. Comme beaucoup des pionniers de la photographie, le problème de la propriété intellectuelle des images pose au photographe de réelles difficultés. Un épisode significatif peut être retracé à la lecture d'une série d'articles parus dans The Graphic en 1878 intitulée An artist pilgrimage to the Holy Land. L'auteur propose un parcours en Terre Sainte illustré par de nombreuses gravures. La plupart d'entre elles sont des copies de vues assez connues de Frank Mason Good ${ }^{66}$. Il faut cependant attendre le deuxième chapitre de ce pèlerinage en image pour que l'auteur véritable des images soit identifié par le journaliste, qui avoue tardivement avoir «omis de reconnaître ses obligations» envers Frank Mason Good ${ }^{67}$. Ce problème d'attribution se pose très tôt pour Frank Mason Good, et cela n'a rien d'exceptionnel pour la photographie commerciale naissante. Les images publiées par Frith ne sont jamais identifiées comme ayant été prises par Good. Quand ce dernier commence à pratiquer la photographie en professionnel, une forme de dépôt légal existe pourtant grâce au Fine Arts copyright, adopté le 29 juillet 1862 par le Parlement. Celui-ci étend la protection du copyright à la photographie. Lauteur doit déposer son œuvre à Stationer's Hall à Londres. Francis Frith sait utiliser cette disposition pour protéger la diffusion de son travail comme

63. Lorenzo Marcy, The sciopticon manual, explaining lantern projections in general, and the sciopticon apparatus in particular: Including magic lantern attachments, experiments, novelties, colored and phototransparencies, mechanical movements, Philadelphia, J.A. Moore, 1877, p. viII. La présence de vues de Palmyre confirme le fait que nombre des plaques étaient des reproductions des négatifs de F. M. Good. 64. Ken Jacobson, Odalisques \& Arabesques... op.cit., p. 71. La gravure, visible à la page 171 du premier volume de la version anglaise de l'ouvrage de Georg Ebers (Egypt: descriptive, historical, and picturesque, Londron, 1880, 2 vol.) est réalisée à partir d'une photographie de moucharabieh réalisée par Good pour le compte de Francis Frith durant son premier voyage. Il s'agit du numéro 1931 de la série publiée par Francis Frith. Le musée Victoria et Albert en possède un exemplaire (cote : E.208:639-1994).

65. C'est le cas de plusieurs gravures du même B. Strassberger. L'une d'entre elles montre l'intérieur de la mosquée Amr ibn al-As et paraît largement inspirée par une autre prise de vue de Good ( ${ }^{\circ} 658$, Mosque of Amer. Old Cairo, conservée au Victoria et Albert Museum, à Londres, cote E.208:622-1994). 66. The Graphic (3 août $1878, \mathrm{n}^{\circ} 453$, p. 17 et $20 ; 10$ août $1878, \mathrm{n}^{\circ} 454$ p. $6 ; 31$ août $1878, \mathrm{n}^{\circ} 457$, p. $21 ; 7$ septembre $1878, \mathrm{n}^{\circ} 458$, p. $10 ; 12$ octobre $1878, \mathrm{n}^{\circ} 463$, p. $17 ; 2$ novembre $1878, \mathrm{n}^{\circ} 466$, p. 17).

67. The Graphic, 10 août 1878 , p.5. 
le montre le revers des vues stéréoscopiques qu'il vendait en France ${ }^{68}$. Un système de signature du négatif et du verso de la carte stéréoscopique assure une forme de complément à ce système de défense de son droit d'auteur. Mais, de façon surprenante, Good n'enregistre partiellement ses photographies qu'en 1894, peut-être de guerre lasse ${ }^{69}$.

Le problème du piratage se pose d'abord pour lui à l'étranger où il lui est plus difficile de faire valoir ses droits. Rien n'empêche un éditeur de procéder à des copies de mauvaise qualité des tirages de Good pour vendre à son compte ses images sans recourir aux négatifs originaux. Le photographe est directement confronté à ce problème comme en témoigne une série nommée Eastern Series. L'éditeur, probablement la firme américaine American Stereoscopic Company, copie des tirages de Good pour réaliser ses propres développements en reprenant jusqu'au nom de la série éditée par Frank Mason Good lui-même [fig. 11 et 12]. Ce type de pratique était facilité par l'absence totale de loi sur les droits d'auteur à une échelle internationale. Le problème est bien connu comme le signale un article du British Journal of Photography: «Dans les pays où il existe une demande forte, ou assez forte, pour de telles productions, où les prix sont quatre fois plus élevés que chez nous et où il n'existe aucune contrainte légale pour éviter qu'on ne copie des plaques britanniques, on ne saurait s'étonner que la tentation de devenir un pirate soit trop forte pour que l'on y résiste. Dans un courrier, M. J. T. Taylor [...] évoque de manière feutrée la possibilité que des vendeurs américains et des producteurs de lanternes magiques et de plaques aient copié des plaques de York et sans doute d'autres productions britanniques. Un autre correspondant, dont on trouvera l'article à la page 143 de ce numéro, est moins réticent, car il affirme qu'alors qu'il résidait aux États-Unis, on lui demanda souvent, en sa qualité d'opérateur photographique, de réaliser des copies de productions anglaises à des fins commerciales. Il comprenait fort bien que de telles copies seraient vendues comme des productions importées aux prix appliqués pour ce type de produits, c'est-à-dire cinq shillings et deux pence pièce. Tant qu'il n'y aura pas de loi internationale sur le droit d'auteur et, peut-on ajouter, aussi longtemps que les taxes à l'importation sur les transparents photographiques seront si élevées, les producteurs anglais vivront sous la menace de voir leur travail copié» ${ }^{70}$.

68. Cf. la série complète conservée à la bibliothèque nationale de France (cote: EK5- 1 FRITH, Francis). 69. Cf. les références COPY 1/418/198 à 204 et COPY 1/418/329 à 331 (National Archives, Kew, Royaume-Uni) qui sont des dépôts légaux pour des prises de vues publiées dans les Universal Series de Frith et des vues d'Espagne.

70. The British Journal of Photography, 'Pirating lantern slides', $\mathrm{n}^{\circ} 1243$, vol. XXXI, 29 février 1884, p. 129-130: "In countries where there is a large or even moderately-large demand for such productions, where the prices are four times in excess of ours, and where there is no legal restraint upon the copying of English slides, it is scarcely to be wondered at if the temptation to become pirates is too great to be successfully withstood. In a letter having a bearing on this subject, from Mr. J. T. Taylor, in our issue of the $15^{\text {th }}$ instant, he dimly hints at the possibility of the copying, by American dealers in and manufacturers of lantern slides and appliances, of York's slides, and presumably others which are of English manufacture. But another correspondent, whose communication will be found on page 143 of this number, 


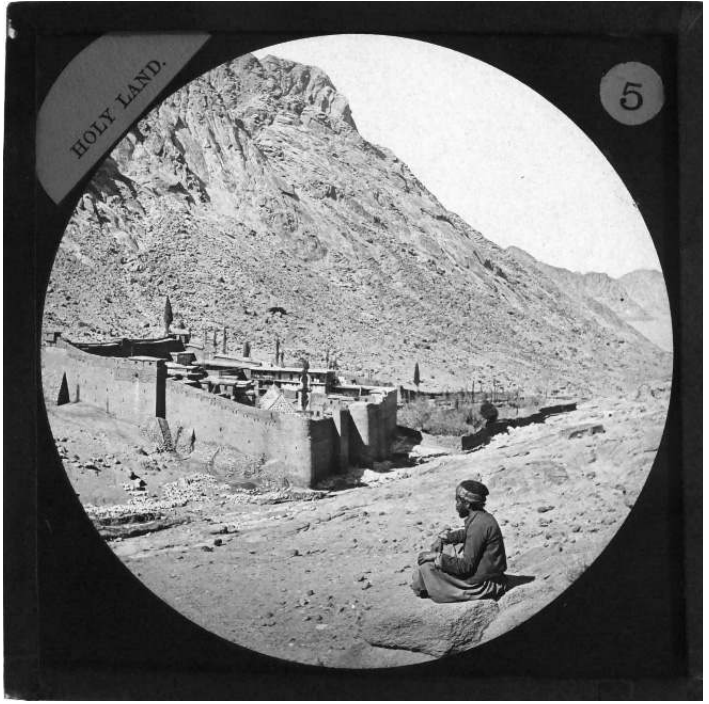

Figure 11. Frank Mason Good, Convent of Sinai at the foot of Mount Horeb, plaque pour lanterne magique, publiée par la firme York and Son dans les années 1880 à partir d'un négatif sur plaque de verre au collodion réalisé à la fin des années 1860 .

L'auteur évoque la firme York qui produisit des plaques de lanterne magique à partir de négatifs de Good. Il mentionne en outre le site de Pétra dont il fut l'un des premiers photographes. Si l'article ne fait pas de référence directe à son œuvre, il permet de comprendre dans quel contexte son imagerie était utilisée et parfois illégalement reproduite ${ }^{71}$. Légale ou non, la diffusion de l'œuvre de Frank Mason Good en Europe ou aux États-Unis témoigne d'une première internationalisation du marché photographique. La photographie de Good, au contexte très britannique, ne reste pas confinée à un splendide isolement. À l'image d'autres studios et éditeurs, comme Francis Frith et plus tard George Washington Wilson ou York and Sons pour les plaques de lanterne magique, le photographe voit ses images consommées aussi bien aux États-Unis qu'en France ou en Allemagne.

En dépit de ses particularismes, la photographie britannique de Palestine domine la production occidentale des années 1850 aux années 1870 aux côtés des travaux français. Elle participe largement à former une première

is less reticent; for he says that when residing in the United States he, as a photographic operator, was often called upon to copy English productions for commercial purposes. It was quite well understood by him that such copies were to be sold as "imported" productions at the prices charged for plain imported slides, namely, five shillings and two pence each. So long as there is no international copyright law to which we may also add that so long as there is such a high protective import duty on photographic transparencies just so long will English makers have to submit to the risk of having their works copied." 71. Les firmes américaines Kilburn (Littleton) et Anthony (New York) ont aussi utilisé des images de Frank Mason Good sans que celles-ci n'évoquent son nom, $c f$. W.S. Clark, Illustrated history, comprising in a condensed form a history of the United States, a geography of the western continent, and the chief objects of interest on the eastern continent, including a hihstorical and descriptive sketch of the Holy Land, by W.S. Clark. illustrated by stereoscopic views published by E. \& H. T. Anthony, New-York, Rockford (Illinois), J. H. Clark, 1870. 


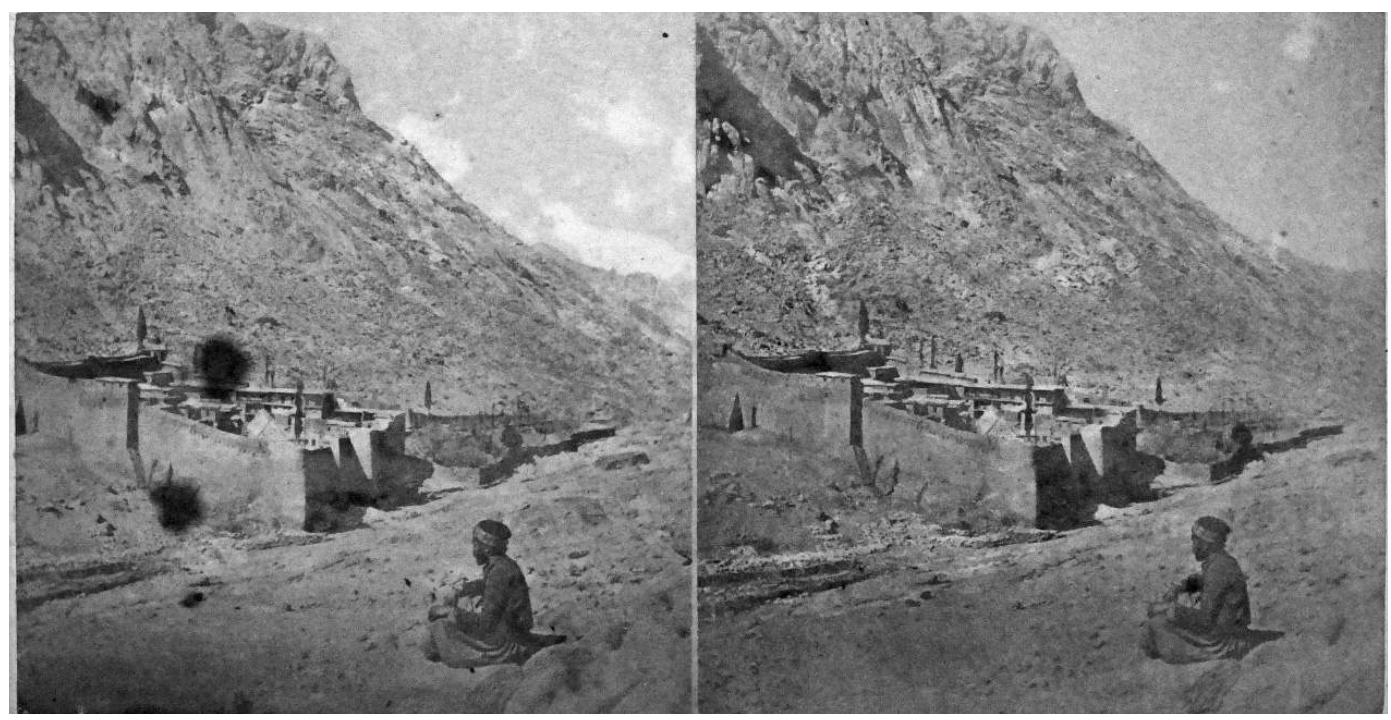

Figure 12. Sinai - Convent of the Sinai at the foot of the Horeb, vue stéréoscopique, copie non-attribuée d'une vue stéréoscopique de Frank Mason Good, éditeur anonyme, années 1870 .

représentation de la Terre Sainte à l'âge de l'image argentique, parfois très marquée par des passions propres au Royaume-Uni. Peu à peu, les photographes résidents, comme Félix Bonfils, Yessayi Garabédian ou, plus tard, ceux de l'American Colony, rénovent à leur tour l'imagerie de cette terre. Il faut attendre les années 1910 pour voir un Britannique jouer à nouveau un rôle déterminant dans l'invention visuelle de la Palestine quand Murray Rosenberg, secrétaire honoraire de la fédération sioniste d'Angleterre, réalise le premier film sur la région ${ }^{72}$.

\section{Daniel Foliard est maître de conférences en civilisation britannique à l'Université Paris-Ouest-Nanterre La Défense.}

